PROCEEDINGS OF THE

AMERICAN MATHEMATICAL SOCIETY

Volume 137, Number 6, June 2009, Pages 2063-2073

S 0002-9939(08)09756-6

Article electronically published on December 15, 2008

\title{
WHEN LOWER ENTROPY IMPLIES STRONGER DEVANEY CHAOS
}

\author{
GRZEGORZ HARAŃCZYK AND DOMINIK KWIETNIAK
}

(Communicated by Jane M. Hawkins)

\begin{abstract}
It is proved that the infimum of the topological entropy of continuous topologically exact interval (circle) maps is strictly smaller than the infimum of the topological entropy of continuous interval (circle) maps, which are topologically mixing, but not exact. Interpreting this result in terms of popular notions of chaos, one may say that on the interval (circle) lower entropy implies stronger Devaney chaos. Moreover, the infimum of the entropy of mixing circle maps is computed. These theorems may be considered as a completion of some results of Alsedà, Kolyada, Llibre, and Snoha (1999).
\end{abstract}

\section{INTRODUCTION}

It is interesting to study lower bounds of the topological entropy in various classes of transitive maps on a given space. This direction of research has a long history and is rich in achievements. In this paper we aim not only to extend the list of spaces and classes of maps for which these infima of topological entropies are known. We also hope that our work will shed some new light on the connection between the topological entropy and the so-called chaotic properties of continuous maps. In particular, we show cases where information that the topological entropy of the system under consideration is sufficiently low implies that the system exhibits stronger chaotic behaviour. This contradicts common intuition which treats topological entropy as a measure of chaos. This paradox is due to a popular belief that the topological entropy is a global and quantitative indicator of the complicated orbit structure of the underlying system. Our results support interpretation of the entropy as a local and qualitative invariant. That is, high entropy may be caused by local erratic behaviour of orbits, and it is positiveness of the entropy, not its exact value, that indicates the complexity of the system.

\section{Preliminaries AND PROBlem Formulation}

2.1. Preliminaries. Let $X$ be a metric space, and let $f$ be a continuous map from $X$ to itself. We assume that the reader is familiar with the basic concepts connected to dynamical systems obtained by iteration of $f$ such as (periodic) orbit, (semi-) conjugacy, etc.; see [3]. To be precise we recall some terminology. In what follows

Received by the editors August 18, 2008.

2000 Mathematics Subject Classification. Primary 37B40, 37B20; Secondary 37E05, 37E10.

The second author was supported in part by the Ministry of Science and Education grant no. N 201272333 for the years 2007-2009.

(C)2008 American Mathematical Society Reverts to public domain 28 years from publication 
$n$ will denote a positive integer, by "a real interval" we mean "a connected subset of the real line with nonempty interior", and "a map" means "a continuous map". The orbit of a periodic point of period $n$ is called an $n$-cycle. A map $f$ is transitive if for every nonempty open subsets $U$ and $V$ of a topological space $X$ there is an $n$ such that $f^{n}(U) \cap V \neq \emptyset$. There are two other, gradually stronger notions: a map $f$ is mixing if for any nonempty sets $U$ and $V$ open in $X$, there is an $N>0$ such that $f^{n}(U) \cap V \neq \emptyset$ for $n \geq N$; a map $f$ is exact if for any nonempty open subset $U$ of $X$ there is an $n$ such that $f^{n}(U)=X$. We say that a map $f$ is pure mixing if $f$ is mixing but not exact. Clearly, exactness implies mixing, and mixing implies transitivity. We recommend [12] as a source for further information on transitivity.

If $X$ is a compact space, then one may define the topological entropy of $f$. The entropy of $f$ is a (possibly infinite) number $h(f) \in[0,+\infty]$. To some extent it measures how complicated the dynamics of $f$ is. For a definition of the entropy we refer the reader to [3]. We will use the basic properties of the entropy such as those in [3, Section 4.1] without further reference.

2.2. Problem formulation. Let $\mathcal{F}$ be a subclass of the class of transitive self-maps of a given compact metric space $X$. By $\inf h(\mathcal{F})$ we understand the best lower bound for the topological entropy of maps from $\mathcal{F}$, that is, $\inf h(\mathcal{F})=\inf \{h(f): f \in \mathcal{F}\}$. Moreover, we say that $\inf h(\mathcal{F})$ is attainable if there exists a map $f \in \mathcal{F}$ such that $h(f)=\inf h(\mathcal{F})$. In this paper we concentrate on the case when the space $X$ is the compact interval $[0,1]$ or the unit circle $\mathbb{S}^{1}=\{z \in \mathbb{C}:|z|=1\}$, and the class $\mathcal{F}$ is a class of transitive continuous self-maps of $X$ or one of its subclasses of mixing, pure mixing, or exact maps.

2.3. Known results. It is known that the best lower bound of topological entropy in the class of transitive maps of $[0,1]$ is $(1 / 2) \log 2$ and is attainable (see [8]). If we restrict ourselves to the class of mixing interval maps 1 then the relevant bound is the same, but it is no more attainable. This is probably well known, but we were unable to localize any source, except [14.

When the underlying space is $\mathbb{S}^{1}$, the infimum is 0 , and it is attainable. Even more can be said if we consider the degree of a circle map. We summarize known infima of the entropy of transitive circle maps in the next theorem. This is a compilation of [1, Theorem 1.3] and [13, Theorem 5]. For more information on original sources for some parts of this result, see 11. Similar facts for various classes of transitive maps on topological graphs can be found in [3, pages 340-343].

Theorem 2.1. Let $f$ be a transitive circle map of degree $d$.

(1) If $|d|>1$, then $h(f) \geq \log |d|$ and there exists an exact circle map $f_{d}$ of degree $d$ such that $h\left(f_{d}\right)=\log |d|$; e.g., take $f_{d}=\left.z^{d}\right|_{\mathbb{S}^{1}}$.

(2) If $d=0$, then $h(f) \geq \log 2$. Moreover, there exists an exact circle map $f_{0}$ of degree 0 such that $h\left(f_{0}\right)=\log 2$.

(3) If $d=1$, then either $f$ has no periodic points and it is semiconjugated to an irrational rotation, and $h(f)=0$, or $f$ has a dense set of periodic points, and $h(f)>0$. Moreover, for every $\varepsilon>0$ there exists an exact circle map $f_{\varepsilon}$ of degree 1 such that $0<h\left(f_{\varepsilon}\right) \leq \varepsilon$.

(4) If $d=-1$, then $h(f) \geq(1 / 2) \log 3$. Moreover, there exists a transitive circle map $f_{-1}$ of degree -1 such that $h\left(f_{-1}\right)=(1 / 2) \log 3$.

\footnotetext{
${ }^{1}$ We adopt a convention that "an interval (a circle) map" means "a continuous self-map of the interval $[0,1]\left(\right.$ of the circle $\left.\mathbb{S}^{1}\right)$ ".
} 


\section{MAin RESUlts}

First, we prove that the infimum of the topological entropy of pure mixing interval maps is greater than the infimum of the entropy of transitive or exact maps.

Theorem A. Let $\mathcal{P}$ denote the class of pure mixing interval maps. Then inf $h(\mathcal{P})=$ $(1 / 2) \log 3$ and is not attainable.

Then, we characterize pure mixing circle maps as those which are factors of pure mixing interval maps, and hence we obtain a similar result about the entropy as above.

Theorem B. Let $\mathcal{P}^{d}$ denote the class of pure mixing circle maps of degree $d$. Then

(1) $\inf h\left(\mathcal{P}^{1}\right)=\log 3$ and is not attainable.

(2) $\inf h\left(\mathcal{P}^{-1}\right)=(1 / 2) \log 3$ and is not attainable.

Finally, we complete the picture presented in Theorem 2.1 and show that the infimum of the entropy of mixing circle maps of degree -1 is equal to $(1 / 2) \log 3$ and is not attainable.

Theorem C. Let $\mathcal{M}^{-1}$ denote the class of mixing circle maps of degree -1 . Then $\inf h\left(\mathcal{M}^{-1}\right)=(1 / 2) \log 3$ and is not attainable.

To obtain our results we find it necessary to refine standard tools used for computation of the entropy of interval and circle maps. We decided to break proofs of main theorems into a sequence of simpler propositions which may be of more general interest; hence we postpone the proofs of the main results until Section 7.

Lower entropy implies stronger chaos. Recall that Devaney [1] proposed a definition of chaos for a map $f$ of an infinite metric space $X$, which is equivalent by [4] to $f$ fullfilling two conditions: $f$ is transitive, and the set of periodic points of $f$ is dense in $X$. On the other hand, exactness and mixing can be regarded as stronger notions of transitivity, the "more chaotic" ones. Since every transitive interval map and every mixing circle map have dense sets of periodic points (see [6], and [10], respectively), for the circle and the compact interval, mixing and exact maps form subclasses of the class of chaotic maps. With the above terminology our results can be reformulated to sound "paradoxical". Let $\mathcal{M}(\mathcal{E}, \mathcal{P})$ denote the class of mixing (exact, pure mixing) interval (circle) maps. We prove that inf $h(\mathcal{P})>\inf h(\mathcal{M})$, that is, adding "more chaotic" maps from the class $\mathcal{E}$ to "less chaotic" maps from $\mathcal{P}$ decreases the possible entropy. For mixing interval (circle) maps sufficiently low entropy implies exactness.

We remark that similar results hold for pure mixing maps on topological graphs, and will be presented in a forthcoming paper. Note that for every compact metric space $X$, we have $h(f)>0$ for any exact map $f: X \mapsto X$ [13] and there is a pure mixing homeomorphism of the Cantor set with a dense set of periodic points and zero topological entropy [15]. This shows that the phenomenon observed for interval and circle maps does not hold in general.

\section{Auxiliary Results}

4.1. Interval maps. Since we need to consider maps not necessarily defined on compact intervals, we have to reformulate some definitions and theorems in this nonstandard context. We hope that our approach will unify and clarify some ideas scattered through the literature of the subject. 
We begin with two propositions which generalize results given for interval maps in [5] and 7]. They may be proved in much the same way as in the original references.

Proposition 4.1. Let $f$ be a transitive map of a real interval $J$. Then exactly one of the following statements holds:

(1) $f^{2}$ is transitive;

(2) there exist intervals $K, L \subset J$, with $K \cap L=\{c\}$ and $K \cup L=J$, such that $c$ is the unique fixed point for $f, f(K)=L$ and $f(L)=K$.

Proposition 4.2. For a map $f$ of a real interval $L$ the following statements are equivalent:

(1) $f^{2}$ is transitive,

(2) $f$ is mixing,

(3) for every interval $J \subset L$ and for any compact interval $K$ contained in the interior of $L$ with respect to the natural topology of the real line there is an $N>0$ such that $K \subset f^{n}(J)$ for $n \geq N$.

The next result characterizes pure mixing interval maps and comes from 9. We say that an endpoint $e \in\{0,1\}$ is accessible for an interval map $f$ if there is an $x \in(0,1)$ such that $f^{n}(x)=e$ for some $n$. An endpoint is inaccessible if it is not accessible.

Proposition 4.3. A mixing interval map $f$ is pure mixing if and only if $f$ has an inaccessible endpoint. Moreover, if an endpoint is inaccessible for $f$, then either it is a fixed point or both endpoints form a two-cycle for $f$. In the latter case the second endpoint is also inaccessible.

4.2. Quasihorseshoes and entropy. We can now formulate the definition of a quasihorseshoe, which is a generalization of the well-known notion of a horseshoe. Then we establish a connection between the entropy and the existence of quasihorseshoes. Although the main ideas are not new, our methods allow more subtle analysis which is needed to show that for some classes of maps $\mathcal{F}$ the infima inf $h(\mathcal{F})$ we are going to compute are not attainable.

Let $f$ be a map from a real interval $L$ to $\mathbb{R}$. An s-quasihorseshoe for $f$ is a compact interval $J \subset L$, and a collection $\mathcal{C}=\left\{A_{1}, \ldots, A_{s}\right\}$ of $s \geq 2$ nonempty compact subsets of $J$ fulfilling the following three conditions: (a) each set $A \in \mathcal{C}$ is a union of a finite number of compact intervals, (b) the interiors of the sets from $\mathcal{C}$ are pairwise disjoint, (c) $J \subset f(A)$ for every $A \in \mathcal{C}$. A quasihorseshoe $(J, \mathcal{C})$ is tight if $J$ is the union of elements of $\mathcal{C}$ and $f(A)=J$ for every $A \in \mathcal{C}$. A quasihorseshoe $(J, \mathcal{C})$ is loose if the union of elements of $\mathcal{C}$ is a proper subset of $J$. An $s$-quasihorseshoe (a tight $s$-quasihorseshoe) $(J, \mathcal{C})$ for $f$ is called an $s$-horseshoe (a tight $s$-horseshoe) if every $A \in \mathcal{C}$ is a compact interval. Our definition of a horseshoe is equivalent to the definition from [3, page 204]. If there is an $s$-quasihorseshoe ( $s$-horseshoe, etc.) $(J, \mathcal{C})$ for $f$, we simply say that $f$ has an $s$-quasihorseshoe (s-horseshoe, etc.), and $J$ carries a quasihorseshoe (s-horseshoe, etc.) for $f$.

It is straightforward to see that the proofs of [3, Lemma 4.3.1] and [3, Proposition 4.3.2] are, with the necessary changes, valid for our quasihorseshoes instead of horseshoes (see also [3, Remark 4.3.4]). For completeness we reformulate Lemma 4.3.1 and Proposition 4.3.2 of [3] as Lemma 4.4 and Proposition 4.5. 
Lemma 4.4. If $(J, \mathcal{C})$ is an s-quasihorseshoe for a map $f$ of a real interval, then for every $n$ there exists an $s^{n}$-quasihorseshoe $(J, \mathcal{D})$ for $f^{n}$ such that each set $A$ in $\mathcal{D}$ is a subset of some $B$ in $\mathcal{C}$.

Proposition 4.5. If an interval map has an s-quasihorseshoe, then $h(f) \geq \log s$.

Lemma 4.6. If $(J, \mathcal{C})$ is an s-quasihorseshoe for a transitive map $f$ of a real interval $L$ and $J \neq L$, then $f$ has a loose s-quasihorseshoe $(J, \mathcal{D})$.

Proof. By transitivity $J$ must be a proper subset of $f(J)$. It is enough to remove from any $B \in \mathcal{C}$ a tiny neighborhood of a point mapped outside $J$.

Lemma 4.7. Assume that $(J, \mathcal{C})$ is a tight s-horseshoe for a transitive interval map $f$. If $f$ has more than $s$ fixed points, then $f$ has a loose s-quasihorseshoe $(J, \mathcal{D})$.

Proof. By Lemma 4.6 we get $J=[0,1]$; hence there is an interval $[u, v] \in \mathcal{C}$ containing two fixed points, say $x$ and $y$ with $x<y$. Since $f$ is transitive, the interval $[x, y]$ cannot be invariant and $\left.f\right|_{[x, y]}$ cannot be strictly monotone. Thus, we can find $a, b \in(x, y)$ such that $f([u, v])=f(A)$, where $A=[u, a] \cup[b, v]$. Now replace $[u, v]$ in $\mathcal{C}$ by $A$.

Proposition 4.8. If a transitive map $f$ of a real interval $L$ has a loose $s$-quasihorseshoe, then there exists $N>0$ such that for every $n \geq N$ the map $f^{n}$ has an $\left(s^{n}+1\right)$-quasihorseshoe. Additionally, if the domain of $f$ is compact, then $h(f)>$ $\log s$.

Proof. Let $(J, \mathcal{C})$ be a loose $s$-quasihorseshoe for $f$. By Propositions 4.2 and 4.1 any transitive map with a quasihorseshoe is mixing. We can find a compact interval $K \subset J$ such that $K \cap A=\emptyset$ for any $A \in \mathcal{C}$. We will find an $N>0$ such that $f^{n}(K) \supset J$ for all $n \geq N$. Then for $n \geq N$ we can take an $s^{n}$-quasihorseshoe $\left(J, \mathcal{D}_{n}\right)$ for $f^{n}$ constructed in Lemma 4.4 and set $\mathcal{D}_{n}^{\prime}=\mathcal{D}_{n} \cup\{K\}$ to prove the first part. Consider the following cases. If $J$ does not contain any endpoint of $L$, then use Proposition 4.2, If $J$ contains an endpoint $e \in L$, then $e \in f(B)$ for some $B \in \mathcal{C}$ such that $e \notin B$. If $B$ contains the other endpoint of $L$, then $f$ is exact, and there is an $N>0$ such that $f^{n}(K)=L$ for $n \geq N$. If $B$ does not contain any endpoints, then by Proposition 4.2 there is an $N^{\prime}>0$ such that $B \subset f^{n}(K)$ for all $n \geq N^{\prime}$. Set $N=N^{\prime}+1$ to see that $J \subset f^{n}(K)$ for every $n \geq N$.

For the proof of the second part, observe that if the domain of $f$ is a compact interval and $n>N$, where $N$ is defined as above, then

$$
h(f)=\frac{1}{n} h\left(f^{n}\right) \geq \frac{1}{n} \log \left(s^{n}+1\right)>\log s,
$$

where the first inequality follows from Proposition 4.5 .

4.3. Circle maps. For the basic facts concerning circle maps we refer the reader to [3, Chapter 3] where one can find a detailed exposition of the theory, including proofs. Here we only set up some notation.

In what follows $e$ denotes the natural projection of $\mathbb{R}$ onto $\mathbb{S}^{1}$ given for $x \in \mathbb{R}$ by $e(x)=\exp (2 \pi i x)$. We write $\operatorname{deg} f=d$ for the degree of a circle map [3, page 109]. We also recall that for a lifting $F$ of a circle map of degree 1 one may define a rotation interval [3, page 148], which is a nonempty compact connected subset of the real line, denoted by $\operatorname{Rot} F$. For each $r \in \operatorname{Rot} F$ there exists $x_{r} \in \mathbb{R}$ for which one may introduce the rotation number of $x$ with respect to $F$ [3] page 135], denoted by $\rho\left(x_{r}\right)$, and $\rho\left(x_{r}\right)=r$. 
Lemma 4.9. Let $F$ be a lifting of a circle map $f$. If $F$ has an s-quasihorseshoe, then $h(f) \geq \log s$. Moreover, if $\left.F\right|_{L}$ is transitive for some compact interval $L$ containing a quasihorseshoe and the quasihorseshoe is loose, then $h(f)>\log s$.

Proof. Let $(J, \mathcal{C})$ be an $s$-quasihorseshoe for $F$. Then the set

$$
X=\left\{x \in J: F^{n}(x) \in J \text { for all } n\right\}
$$

is nonempty, compact, and invariant for $F$. It is well known that $h\left(\left.F\right|_{X}\right) \geq \log s$. Now, $e(X)$ is an $f$-invariant subset of the circle, and $\left.f\right|_{e(X)}$ is a factor of $\left.F\right|_{X}$ through $\left.e\right|_{X}$. As preimages of points in the circle through $\left.e\right|_{X}$ have bounded finite cardinality, we conclude that $h\left(\left.f\right|_{e(X)}\right)=h\left(\left.F\right|_{X}\right)$. The second part of this lemma is obvious, by the above considerations and the proof of Proposition 4.8 .

We use the characterization of pure mixing circle maps, which can be found in [10. Lemma 5.2.]. Note that here we use a slightly different terminology. We say that a point $x \in \mathbb{S}^{1}$ is accessible for a circle map $f$ if for every compact interval $K \subset \mathbb{S}^{1} \backslash\{x\}$ there is an $n$ such that $x \in \operatorname{int} f^{n}(K)$. A point which is not accessible is called inaccessible.

Proposition 4.10. A mixing circle map $f$ is pure mixing if and only if there is an inaccessible point. If an inaccessible point exists, then it is unique and fixed for $f$.

The next result can be easily derived from the proof of [10, Lemma 5.2].

Corollary 1. Let $f$ be a pure mixing circle map and let $c$ be the unique inaccessible fixed point for $f$. Then:

(1) For every pair of compact subintervals $K$ and $L$ of $\mathbb{S}^{1} \backslash\{c\}$ there is an $N>0$ such that $K \subset f^{n}(L)$ for all $n \geq N$.

(2) There exist an interval of the form $[C, C+1] \subset \mathbb{R}$ and a pure mixing map $F$ from $[C, C+1]$ into itself such that $e \circ F=f \circ e$ and $e(C)=e(C+1)=c$.

(3) The degree of $f$ is equal to 1 , or -1 , and $\operatorname{Rot}\left(F^{2}\right)=\{0\}$ for every lifting $F$ of $f$.

Proposition 4.11. Let $F$ be a lifting of the mixing circle map $f$ of degree one. If Rot $F=[\alpha, \beta]$ for some $\alpha<0<\beta$, then $F$ is a mixing map of the real line.

Proof. Fix any compact interval $K \subset \mathbb{R}$. Choose positive integers $p, q, s$ and $t$ such that $\alpha \leq-p / q<0$ and $0<s / t \leq \beta$. By [3, Theorem 3.7.20] there are periodic points $x$ and $y$ for $f$ such that $\rho(X)=-p / q$ and $\rho(Y)=s / t$ for every $X \in e^{-1}(x)$ and $Y \in e^{-1}(y)$.

By Proposition 4.2 it is enough to show that

$$
\lim _{m \rightarrow \infty}\left(\inf F^{m}(K)\right)=-\infty \text { and } \lim _{m \rightarrow \infty}\left(\sup F^{m}(K)\right)=+\infty .
$$

It follows from Corollary 1 that $f$ is exact. Hence, there exists $n$ such that $F^{n}(K)$ contains an interval of the form $[Z, Z+1]$ for some $Z \in \mathbb{R}$. Then, by [3, Proposition 3.2.2] the set $F^{n}(K)$ contains some points $X_{0}$ and $Y_{0}$ such that $e\left(X_{0}\right)=x$, and $e\left(Y_{0}\right)=y$. Using [3, Lemma 3.7.2], we see that $F^{q j}\left(X_{0}\right)=X_{0}-p j$ and $F^{s j}\left(Y_{0}\right)=Y_{0}+t j$ for every $j>0$. This implies the condition given by (4.1). 


\section{LOWER BOUNDS}

The lower bounds for the entropy in Theorems A and B are corollaries of the two facts we will prove in this section.

Lemma 5.1. Assume that $f$ is a pure mixing interval map.

(1) If there is a nonaccessible fixed point, then $h(f)>\log 3$.

(2) If the endpoints of $[0,1]$ form a two-cycle, then $h(f)>(1 / 2) \log 3$.

Proof. By transitivity of $f^{2}$ and the equality $h\left(f^{2}\right)=2 h(f)$, we need to prove only the first assertion. Hence, without loss of generality, we assume that $f^{-1}(0)=\{0\}$. By Proposition 4.8 it is enough to show that $f$ has a loose 3-horseshoe.

By [2, Proposition 4.2] $f$ has a 3 -horseshoe $(J, \mathcal{C})$, but since 0 is inaccessible, $0 \notin J$; hence $J \neq[0,1]$. We apply Lemma 4.6 to finish the proof.

Proposition 5.2. If $f$ is a mixing circle map of degree -1 , then $h(f)>(1 / 2) \log 3$.

Proof. Let $F$ be a lifting of $f$. Then $F^{2}$ is a lifting of the circle map $f^{2}$ of degree 1 . By [1, Lemma 4.4], we can distinguish two cases, according as the rotation interval Rot $F^{2}$ of $F^{2}$ is of the form $[-a, a]$ for some $a>0$, or $\operatorname{Rot} F^{2}=\{0\}$.

We first consider the case when Rot $F^{2}=[-a, a]$ for some $a>0$. Then 0 is an interior point of Rot $F^{2}$, and by Proposition 4.11 we conclude that $F^{2}$ is a mixing map of the real line. It follows from [3, Remark 3.9.2] that $F^{2}$ has a 3 -horseshoe. By Lemma 4.6 every horseshoe for a mixing real map must be loose. Moreover, Lemma 4.9 shows that $h\left(f^{2}\right)>\log 3$, and the proof for the first case is done.

For the remaining case assume that $\operatorname{Rot} F^{2}=\{0\}$. Arguing as in the proof of [1, Proposition 4.8] we find a point $C \in \mathbb{R}$ such that $F^{2}(C)=C, F^{2}(C+1)=C+1$, and $F^{2}([C, C+1])=[C, C+1]$. Moreover, by [1, Proposition 4.5] the map $\left.F^{2}\right|_{[C, C+1]}$ has a 3 -horseshoe. Clearly, $\left.F^{2}\right|_{[C, C+1]}$ is mixing.

We claim that $\left.F^{2}\right|_{[C, C+1]}$ has at least four fixed points in $(C, C+1)$. It follows from [3, Theorem 3.3.5] that there exist two points $A, B \in[C, C+1)$, such that $e(A)$ and $e(B)$ are fixed for $f$. Since the rotation interval of $F^{2}$ is degenerate, $A$ and $B$ are fixed points for $F^{2}$. Since $f$ is transitive and of $\operatorname{deg} f=-1$, the periodic points of $f$ are dense in $\mathbb{S}^{1}$. If all periodic points of $f$ were fixed, then $f$ would be an identity map. Therefore $f$ has an $n$-cycle with $n>1$; hence $f$ has a two-cycle $\{x, f(x)\}$ (see [3. Theorem 3.6.8]). It follows from [3, Proposition 3.2.2] that there are points $X$ and $Y$ in $[C, C+1)$ such that $e(X)=x$ and $e(Y)=f(x)$. Clearly, $F^{2}(X)=X$ and $F^{2}(Y)=Y$. Moreover, $\{A, B\} \cap\{X, Y\}$ is empty. This proves the existence of at least four fixed points for $F^{2}$ in $[C, C+1)$, so our claim follows. By Lemma 4.7 our 3-horseshoe is loose. Then $h\left(\left.F^{2}\right|_{[C, C+1]}\right)>\log 3$ by Lemma 4.9.

\section{EXAmples}

We construct examples of mixing (exact) interval and circle maps with entropies arbitrarily close to the bounds obtained in Lemma 5.1 and Proposition 5.2.

Proposition 6.1. For every $\varepsilon>0$ there exists

(1) an exact circle map $f_{1}$ of degree -1 such that $h\left(f_{1}\right) \leq(1 / 2) \log 3+\varepsilon$,

(2) a pure mixing interval map $f_{2}$ such that $h\left(f_{2}\right) \leq(1 / 2) \log 3+\varepsilon$,

(3) a pure mixing interval map $f_{3}$ such that $h\left(f_{3}\right) \leq \log 3+\varepsilon$,

(4) a pure mixing circle map $f_{4}$ of degree -1 such that $h\left(f_{4}\right) \leq(1 / 2) \log 3+\varepsilon$,

(5) a pure mixing circle map $f_{5}$ of degree 1 such that $h\left(f_{5}\right) \leq \log 3+\varepsilon$. 


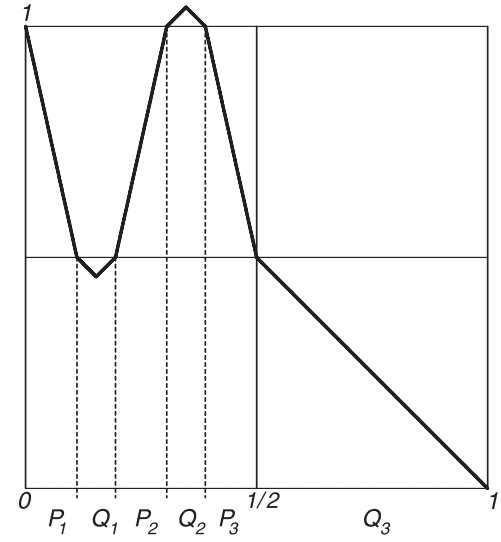

Figure 1. Plot of $\varphi$.

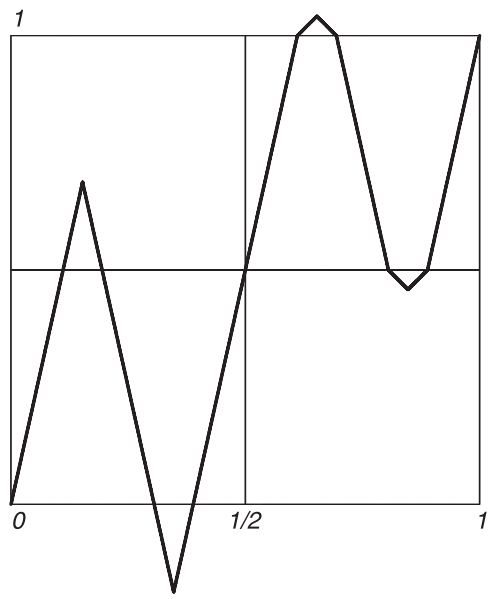

Figure 2. Plot of $\left.\varphi^{2}\right|_{[0,1]}$.

Proof. We begin by constructing an example for (1). For the rest of this section fix $\varepsilon>0$ and $\lambda>3$ such that $\log \lambda<\log 3+\varepsilon$.

Let $\varphi:[0,1] \mapsto \mathbb{R}$ be a map given by a formula

$$
\varphi(x)=\left\{\begin{array}{rccccc}
-\lambda x & + & 1, & 0 & \leq x< & 1 / 2 \lambda ; \\
-x & + & (\lambda+1) / 2 \lambda, & 1 / 2 \lambda & \leq x< & (\lambda+1) / 8 \lambda \\
x & + & (\lambda+1) / 4 \lambda, & (\lambda+1) / 8 \lambda & \leq x< & (\lambda-1) / 4 \lambda \\
\lambda x & + & (3-\lambda) / 4 \lambda, & (\lambda-1) / 4 \lambda & \leq x< & (\lambda+1) / 4 \lambda \\
x & + & (3 \lambda-1) / 4 \lambda, & (\lambda+1) / 4 \lambda & \leq x< & (3 \lambda-1) / 8 \lambda \\
-x & + & (3 \lambda-1) / 2 \lambda, & (3 \lambda-1) / 8 \lambda & \leq x< & (\lambda-1) / 2 \lambda ; \\
-\lambda x & + & (\lambda+1) / 2, & (\lambda-1 / 2 \lambda & \leq x< & 1 / 2 ; \\
-x & + & 1, & 1 / 2 & \leq x \leq & 1 .
\end{array}\right.
$$

We will need additional notation (see Figure 1). Let $p_{1}=1 / 2 \lambda, p_{2}=(\lambda+1) / 8 \lambda$, $p_{3}=(\lambda-1) / 4 \lambda, p_{4}=(\lambda+1) / 4 \lambda, p_{5}=(3 \lambda-1) / 8 \lambda, p_{6}=(\lambda-1) / 2 \lambda$, and $P_{1}=\left[0, p_{1}\right]$, $Q_{1}=\left[p_{1}, p_{3}\right], P_{2}=\left[p_{3}, p_{4}\right], Q_{2}=\left[p_{4}, p_{6}\right], P_{3}=\left[p_{6}, 1 / 2\right], Q_{3}=[1 / 2,1]$.

Observe that $\varphi$ has slope $\pm \lambda$ on the intervals $P_{1}, P_{2}, P_{3}$ and slope \pm 1 on the other intervals of monotonicity. We can extend $\varphi$ to a map $F: \mathbb{R} \mapsto \mathbb{R}$ by defining

$$
F(x)=\varphi(x-\lfloor x\rfloor)-\lfloor x\rfloor,
$$

where $\lfloor x\rfloor$ denotes the integer part of a function. Clearly, $F$ is a lifting of a circle map $f$ of degree -1 . Moreover, it is easy to see that $F$ and $F^{2}$ are Lipschitz with constant $\lambda$ (see Figure 2). It follows that $h(f) \leq(1 / 2) \log \lambda<(1 / 2) \log 3+\varepsilon$. To finish the proof it is now sufficient to show that $f$ is exact. Actually, we will prove a stronger fact:

$$
\lim _{m \rightarrow \infty}\left(\inf F^{m}(K)\right)=-\infty \text { and } \lim _{m \rightarrow \infty}\left(\sup F^{m}(K)\right)=+\infty
$$

for every compact interval $K \subset \mathbb{R}$. We begin by introducing some notation: for $k \in \mathbb{Z}$, let $[a, b]+k$ denote the translate of $[a, b]$ by $k$. Assume that $K \subset[0,1]+k$ for some $k \in \mathbb{Z}$. Observe that, for $\varepsilon$ small enough, $F\left(Q_{1}+k\right) \subset P_{3}-k, F\left(Q_{2}+k\right) \subset$ $P_{1}-k+1$, and $F\left(Q_{3}+k\right) \subset[0,1 / 2]-k$. Combining these inclusions with the fact that for any interval $L$ contained in the set $\left(P_{1} \cup P_{2} \cup P_{3}\right)+k$ the length of the 


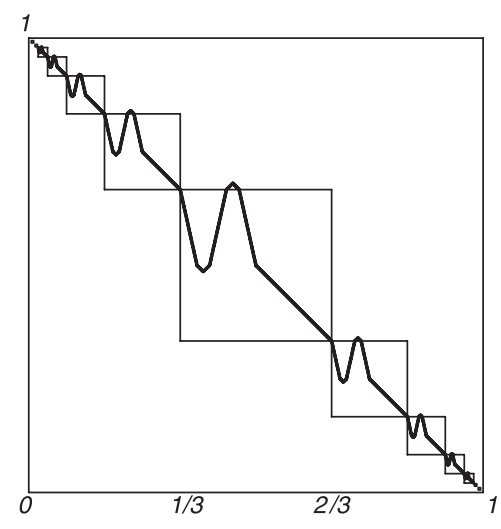

Figure 3. Plot of $\Phi$.

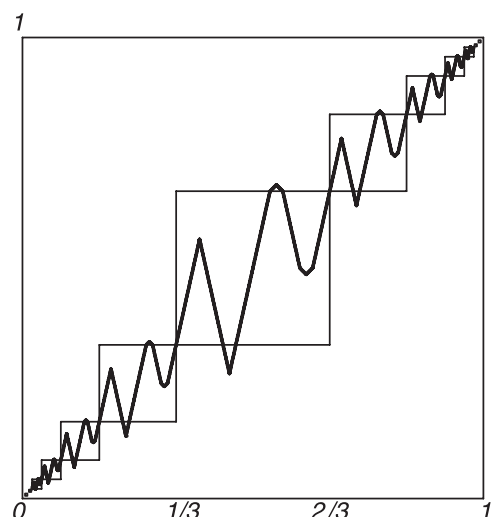

Figure 4. Plot of $\Phi^{2}$.

interval $F(L)$ is at least triple the length of $L$, we see that the length of $F^{n}(K)$ grows and there is some $N$ such that $F^{N}(K) \supset([0,1] \pm k) \cup F\left(Q_{2} \pm k\right)$. Now, a simple induction argument finishes the proof of our claim. The exactness of $f$ follows.

To prove (2), divide the interval $(0,1)$ into intervals $\left\{I_{k}\right\}_{k=-\infty}^{+\infty}$ (overlapping at the endpoints), where $I_{0}=[1 / 3,2 / 3]$ and

$$
\begin{array}{cc}
I_{k}= & {\left[1-\left(3 \cdot 2^{k-1}\right)^{-1}, 1-\left(3 \cdot 2^{k}\right)^{-1}\right], \text { for } k>0,} \\
I_{k}= & {\left[\left(3 \cdot 2^{|k|}\right)^{-1},\left(3 \cdot 2^{|k|-1}\right)^{-1}\right], \text { for } k<0 .}
\end{array}
$$

Let $h_{k}$ denote an affine, orientation-preserving homeomorphism which maps $[0,1]$ onto $I_{k}$. Given $k \in \mathbb{Z}$, we define a map $\Phi:[0,1] \mapsto[0,1]$ on $I_{k}$ as a suitably rescaled map $\varphi$. By "suitably rescaled" we mean that $\left.\Phi\right|_{I_{k}}=h_{-k} \circ \varphi \circ h_{k}^{-1}$. At the endpoints we set $\Phi(0)=1$, and $\Phi(1)=0$. This yields a well-defined interval map (see Figure 3). Observe that $\left.\Phi\right|_{(0,1)}$ is conjugated (via a piecewise affine homeomorphism) to a map $F: \mathbb{R} \mapsto \mathbb{R}$ defined above. Hence, by (6.2) and Proposition 4.2, we see that $\Phi$ is a pure mixing interval map. Moreover, the Lipschitz constant for $\Phi^{2}$ is not greater than $\lambda$ (see Figure 4); hence $h(\Phi) \leq(1 / 2) \log 3+\varepsilon$. Now, take $f_{2}=\Phi$. For the proof of (3) it is enough to define $f_{3}=\Phi^{2}$. To prove (4) and (5) we extend the interval map $\Phi\left(\Phi^{2}\right)$ to a lifting $F_{4}\left(F_{5}\right)$ of a circle map $f_{4}\left(f_{5}\right.$, respectively), the same way as we did in (6.1). Note that $F_{4}$ is degree -1 lifting and $F_{5}$ is of degree 1 . From the above considerations it is clear that the circle maps $f_{4}$ and $f_{5}$ fulfill the conditions given in (4) and (5).

\section{Proofs of the MaIn theorems}

Theorem A. Let $\mathcal{P}$ denote the class of pure mixing interval maps. Then $\inf h(\mathcal{P})=$ $(1 / 2) \log 3$ and is not attainable.

Proof. This is a simple consequence of Lemma 5.1 and Proposition 6.1(2). 
Theorem B. Let $\mathcal{P}^{d}$ denote the class of pure mixing circle maps of degree $d$. Then

(1) $\inf h\left(\mathcal{P}^{1}\right)=\log 3$ and is not attainable.

(2) $\inf h\left(\mathcal{P}^{-1}\right)=(1 / 2) \log 3$ and is not attainable.

Proof. Let $f$ be a pure mixing circle map. We apply Corollary 11 to see that (i) $|\operatorname{deg} f|=1$, (ii) there exists $c$ the unique inaccessible fixed point of $f$, (iii) there exist an interval $J=[C, C+1]$ and a pure mixing map $F$ of $J$ such that $e \circ F=f \circ e$ and $e(C)=e(C+1)=c$. It is clear that $F(C)=C+1$ and $F(C+1)=C(F(C)=C$ and $F(C+1)=C+1)$ if and only if $\operatorname{deg} f=-1(\operatorname{deg}(f)=1)$. If $\operatorname{deg} f=-1$, then $C$ and $C+1$ are inaccessible for $F$. If $\operatorname{deg} f=1$, then $C$ or $C+1$ is inaccessible for $F$. Now apply Lemma 5.1 and the fact that $e$ is at most two-to-one semiconjugacy between $F$ and $f$ to obtain lower bounds for the topological entropy. To show that these bounds are infima, use Proposition 6.1(4) and Proposition 6.1(5).

Theorem C. Let $\mathcal{M}^{-1}$ denote the class of mixing circle maps of degree -1 . Then $\inf h\left(\mathcal{M}^{-1}\right)=(1 / 2) \log 3$ and is not attainable.

Proof. Use Proposition 5.2 and Proposition 6.1(1).

\section{ACKNOWLEDGEMENTS}

Both authors want to express their gratitude to Jozef Bobok, Michał Misiurewicz, and Wojciech Słomczyński for stimulating conversations, and to the anonymous referee for several comments that improved the paper.

\section{REFERENCES}

[1] Ll. Alsedà, S. Kolyada, J. Llibre, and L. Snoha, Entropy and periodic points for transitive maps, Trans. Amer. Math. Soc. 351 (1999), no. 4, 1551-1573. MR1451592 (99f:58117)

[2] Lluis Alsedà, Stewart Baldwin, Jaume Llibre, and Michał Misiurewicz, Entropy of transitive tree maps, Topology 36 (1997), no. 2, 519-532. MR.1415604 (98f:54031)

[3] Lluís Alsedà, Jaume Llibre, and Michał Misiurewicz, Combinatorial dynamics and entropy in dimension one, second ed., World Scientific, River Edge, NJ, 2000. MR.1807264|(2001j:37073)

[4] J. Banks, J. Brooks, G. Cairns, G. Davis, and P. Stacey, On Devaney's definition of chaos, Amer. Math. Monthly 99 (1992), no. 4, 332-334. MR.1157223 (93d:54059)

[5] Marcy Barge and Joe Martin, Chaos, periodicity, and snakelike continua, Trans. Amer. Math. Soc. 289 (1985), no. 1, 355-365. MR779069 (86h:58079)

[6] _ Dense periodicity on the interval, Proc. Amer. Math. Soc. 94 (1985), no. 4, 731-735. MR792293 (87b:58068)

[7] Dense orbits on the interval, Michigan Math. J. 34 (1987), no. 1, 3-11. MR873014 (88c:58031)

[8] A. M. Blokh, Sensitive mappings of an interval, Uspekhi Mat. Nauk 37 (1982), no. 2(224), 189-190. MR650765 (83k:58053)

[9] Alexander M. Blokh, The "spectral" decomposition for one-dimensional maps, Dynamics Report. Expositions Dynam. Systems (N.S.) 4, Springer, Berlin, 1995, pp. 1-59. MR1346496 (96e:58087)

[10] Ethan M. Coven and Irene Mulvey, Transitivity and the centre for maps of the circle, Ergodic Theory Dynam. Systems 6 (1986), no. 1, 1-8. MR837972 (87j:58074)

[11] Robert L. Devaney, An introduction to chaotic dynamical systems, Westview Press, Boulder, CO, 2003. MR1979140(2004e:37001)

[12] Sergir Kolyada and L'ubomír Snoha, Some aspects of topological transitivity-a survey, Grazer Math. Ber., vol. 334, Karl-Franzens-Univ. Graz, Graz, 1997, pp. 3-35. MR.1644768

[13] Dominik Kwietniak and Michał Misiurewicz, Exact Devaney chaos and entropy, Qual. Theory Dyn. Syst. 6 (2005), no. 1, 169-179. MR2273492(2007i:37031) 
[14] Sylvie Ruette, Chaos for continuous interval maps, preprint, available at http://www.math. u-psud.fr/ ruette/publications.html

[15] Benjamin Weiss, Topological transitivity and ergodic measures, Math. Systems Theory 5 (1971), 71-75. MR0296928(45:5987)

Institute of Mathematics, Jagiellonian University, Łojasiewicza 6, 30-348 Kraków, POLAND

E-mail address: gharanczyk@gmail.com

Institute of Mathematics, Jagiellonian University, Łojasiewicza 6, 30-348 Kraków, POLAND

E-mail address: dominik.kwietniak@gmail.com 